\title{
Improving health and carbon footprints of European diets using a benchmarking approach
}

\author{
Elly Mertens ${ }^{1, *}$ (1), Anneleen Kuijsten ${ }^{1,2}$, Argyris Kanellopoulos ${ }^{3}$ (1), Marcela Dofková ${ }^{4}$, \\ Lorenza Mistura ${ }^{5}$, Laura D’Addezio ${ }^{5}$, Aida Turrini ${ }^{5}$, Carine Dubuisson ${ }^{6}$, \\ Sabrina Havard ${ }^{6}$, Ellen Trolle ${ }^{7}$, Marion Eckl ${ }^{1}$, Sander Biesbroek ${ }^{1}$, Jacqueline Bloemhof ${ }^{3, \dagger}$, \\ Johanna $M$ Geleijnse ${ }^{1,2}$ and Pieter van ' $t$ Veer ${ }^{1,2}$ \\ 'Division of Human Nutrition and Health, Wageningen University, P.O. Box 17, 6700 AA Wageningen, the \\ Netherlands: ${ }^{2} \mathrm{TiFN}$, Wageningen University, Wageningen, the Netherlands: ${ }^{3}$ Operations Research and Logistics \\ Group, Wageningen University, Wageningen, the Netherlands: ${ }^{4}$ Center for Health, Nutrition and Food, National \\ Institute of Public Health, Brno, Czech Republic: ${ }^{5}$ Council for Agricultural Research and Economics, Research Centre for \\ Food and Nutrition, Rome, Italy: ${ }^{6}$ Risk Assessment Department, French Agency for Food, Environmental and \\ Occupational Health \& Safety (Anses), Maisons-Alfort Cedex, France: ${ }^{7}$ Division of Risk Assessment and Nutrition, \\ National Food Institute, Technical University of Denmark, Søborg, Denmark
}

Submitted 25 February 2020: Final revision received 21 July 2020: Accepted 21 August 2020: First published online 23 September 2020

\begin{abstract}
Objective: This study aimed to identify diets with improved nutrient quality and environmental impact within the boundaries of dietary practices.

Design: We used Data Envelopment Analysis to benchmark diets for improved adherence to food-based dietary guidelines (FBDG). We then optimised these diets for dietary preferences, nutrient quality and environmental impact. Diets were evaluated using the Nutrient Rich Diet score (NRD15.3), diet-related greenhouse gas emission (GHGE) and a diet similarity index that quantified the proportion of food intake that remained similar as compared with the observed diet. Setting: National dietary surveys of four European countries (Denmark, Czech Republic, Italy and France).

Subjects: Approximately 6500 adults, aged 18-64 years.

Results: When dietary preferences were prioritised, NRD 15.3 was $~ 6 \%$ higher, GHGE was $\sim 4 \%$ lower and $\sim 85 \%$ of food intake remained similar. This diet had higher amounts of fruit, vegetables and whole grains than the observed diet. When nutrient quality was prioritised, NRD15.3 was 16\% higher, GHGE was 3\% lower and $\sim 72 \%$ of food intake remained similar. This diet had higher amounts of legumes and fish and lower amounts of sweetened and alcoholic beverages. Finally, when environmental impact was prioritised, NRD15.3 was 9\% higher, GHGE was $\sim 21 \%$ lower and $\sim 73 \%$ of food intake remained similar. In this diet, red and processed meat partly shifted to either eggs, poultry, fish or dairy.

Conclusions: Benchmark modelling can generate diets with improved adherence to FBDG within the boundaries of dietary practices, but fully maximising health and minimising GHGE cannot be achieved simultaneously.
\end{abstract}

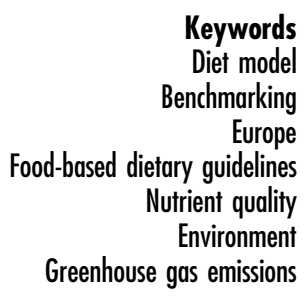

In Europe, unhealthy diets, including overconsumption, contribute to a substantial rise in the incidence of obesity and non-communicable diseases, including CHD, type 2 diabetes and cancer ${ }^{(1)}$. Diets not only impact human health but also the environment ${ }^{(2-4)}$. Hence, there is an urgent need to shift towards healthier and environmentally sustainable diets. Such diets would fulfil nutritional Deceased. requirements, reduce overall disease risk and can be produced within planetary boundaries. In order to find the best balance between the health and environmental dimensions of a diet, mathematical modelling and optimisation techniques are often employed ${ }^{(5-7)}$.

Various models have been developed to optimise diets using individual-level data from specific countries and with specified objectives for health and the environment ${ }^{(8,9)}$. 
Generally, these diet models have taken the form of linear programming and started from a set of food items from dietary surveys with the goal of composing a total diet that satisfies a predefined set of norms for nutritional requirements and environmental footprints. As these models are based on single and unrelated food items, additional constraints are needed to account for cultural acceptance and dietary preferences of the optimised diet $^{(6,10)}$. Examples include the minimum and maximum amount of foods consumed; the associations among foods in meals, such as breakfast cereal and milk and bread and jam and the popularity of foods by minimising deviations from the observed average diet ${ }^{(11)}$.

Recently, Kanellopoulos et al. (2020) ${ }^{(12)}$ presented Data Envelopment Analysis (DEA) as a benchmarking approach that models new diets as a linear combination of observed diets, which implicitly maintains the basic interrelationships among food items in the diet of the study population. Such an approach allows diets from different countries to be modelled in a comparable way and accounts for cultural acceptance and dietary preferences without specifying additional constraints for each country. The present study applies this benchmarking approach to individual-level food consumption data from four European countries, i.e. Denmark, Czech Republic, Italy and France. For men and women in each country, trade-offs are addressed between nutrient quality, environmental impact and dietary preferences. By providing solutions within the range of existing diets, such benchmark models could be useful to guide policies towards healthier and more environmentally sustainable diets that are culturally acceptable for each country and contribute to health and environmental sustainability goals at both the national and European level.

\section{Methods}

\section{Study population and food intake data}

Food consumption data for the adult population, aged 18-64 years, were obtained from nationally representative dietary surveys in four countries: including DANSDA (2005-2008) in Denmark, based on 7-d diet records on consecutive days ${ }^{(13)}$; SISP04 (2003-2004) in the Czech Republic, based on two 24-hour recalls spaced over 3-5 months ${ }^{(14)}$; INRAN-SCAI (2005-2006) in Italy, based on 3-d diet records on consecutive days ${ }^{(15)}$ and INCA-2 Study (2006-2007) in France, based on 7-d diet records on consecutive days ${ }^{(16)}$. In order to enable fair crosscountry comparisons, we sampled two non-consecutive days for each country ${ }^{(17)}$.

Food intakes were classified for each country according to the FoodEx2 classification developed by the European Food Safety Authority ${ }^{(18,19)}$. Nutrient composition of the consumed foods was estimated using country-specific food composition databases ${ }^{(20-26)}$. As explained in our previous publication ${ }^{(27)}$, estimates of greenhouse gas emission
(GHGE, in $\mathrm{kgCO}_{2}$ equivalents $\left(\mathrm{kgCO}_{2} \mathrm{eq}\right) / \mathrm{kg}$ food as eaten) were first obtained from a standardised life-cycle assessment database of GHGE values (SHARP-Indicator Database ${ }^{(28)}$ ). GHGE was then assigned to each of the 944 FoodEx2-codes that were consumed in the four countries.

Quantities of foods were calculated for each individual using the mean of $2 \mathrm{~d}$ and were expressed per $10 \cdot 460 \mathrm{MJ}$ $(2500 \mathrm{kcal})$ for men and per $8.368 \mathrm{MJ}$ (2000 kcal) for women $^{(29)}$. In this way, we accounted for the observed variation in the amounts of foods consumed for different levels of reported energy intake, while maintaining the composition of the diet. Under- and over-reporters were excluded using the Goldberg equation ${ }^{(30)}$ as adopted by Black $^{(31)}$, i.e. cut-off value of 0.96 and 2.49 for a ratio of reported to energy requirement. The present study was conducted on a final sample of diets of 1385 adults in Denmark, 1386 adults in the Czech Republic, 1978 adults in Italy and 1713 adults in France.

\section{The benchmark diet model}

As described by Kanellopoulos et al. ${ }^{(12)}$, the DEA model was used for modelling diets based on nutritional benchmarking that starts from observed diets in a population sample. Differently from Kanellopoulos ${ }^{(12)}$, we specified food groups rather than nutrient intakes as dietary components to in- or decrease in order to arrive at a healthier diet. Thus, the present DEA model uses food-based dietary guidelines (FBDG, see Table 1) as an a priori defined set of guidelines that accounts for the integrated evidence on diet-related disease risk and nutritional adequacy. For each of the observed diets, the model identifies a number of peers with a so-called efficient diet that performs better with respect to the FBDG, i.e. basically with a higher ratio of 'dietary components to increase' to 'dietary components to decrease'. This identification of efficient diets was solved in two stages ${ }^{(12,32)}$, using Xpress-IVE release $1 \cdot 24$. Subsequently, for each subject healthier diets are calculated as a linear combination of the efficient diets of his/her peers. In this modelling step, these 'healthier' linear combinations were constructed in a way to remain as close as possible to the observed diet (MaxP, most preferable), are the healthiest $(\operatorname{maxH}$, highest nutrient density score) or the most environmentally sustainable (MaxS, lowest GHGE). Diets were modelled for each country and for men and women separately.

\section{Model variables and identification of efficient diets}

The energy-standardised survey data were used as the set of observed diets from which efficient diets were identified. For the variable selection, foods were classified into food groups that correspond to health-based FBDG based on available scientific evidence for diets to reduce noncommunicable disease risk factors in the four countries ${ }^{(17)}$. Instead of oils, we included unsaturated fats to increase and saturated fats to decrease. For alcoholic drinks, we used 
Table 1 Dietary components to identify existing healthier diets while benchmarking diets, including capping values if necessary*

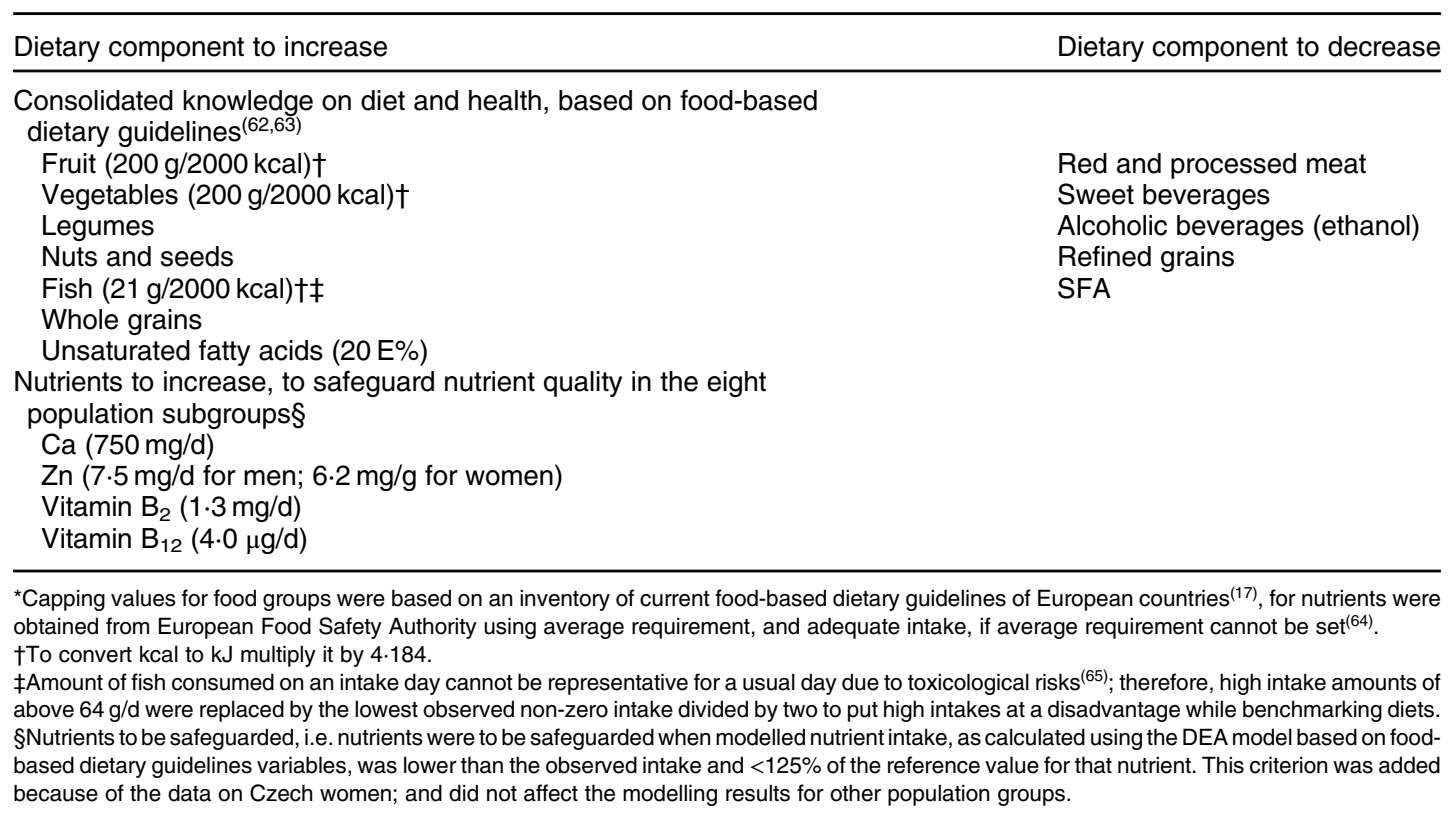

calculated ethanol intake. After including these FBDGbased variables in the model, we subsequently identified and added nutrients that needed to be safeguarded. These were defined as nutrients for which the modelled mean intake after improving adherence to FBDG was lower than the observed intake and was $<125 \%$ of the reference value for that nutrient (Table 1).

For each observed diet and all dietary components included (as mentioned in Table 1), the DEA model compares and weighs the multidimensional ratio of 'dietary components to increase' to 'dietary components to decrease'. As this decision variable is essentially based on ratios, zero intakes are not permitted. Therefore, zero intakes of food groups were replaced by the observed lowest non-zero intake of that food group divided by two ${ }^{(33)}$. Similar to the calculation of the Nutrient Rich Diet score $^{(34,35)}$, the amount consumed for certain food groups and nutrient intakes were capped if higher intakes were not considered to provide additional health benefits (Table 1). For example, consuming up to $200 \mathrm{~g}$ of fruit per $8.368 \mathrm{MJ}$ (2000 kcal) was considered linearly related to increased benefits, whereas no additional benefits were attributed to consuming $>200 \mathrm{~g}$ per $8.368 \mathrm{MJ}$ (2000 kcal). For the food groups in which higher intakes above a certain level are considered harmful, such as fish (see Table 1), observed intakes higher than this level were replaced by the lowest observed non-zero intake divided by two. This ensured that these diets are less likely to be included as a benchmark.

\section{Modelled diets and trade-offs}

For each inefficient diet in the observed data, an alternative healthier diet was modelled as a linear combination of the existing efficient diets and served as the benchmark for the inefficient diet. However, by taking different linear combinations, more options for dietary improvement were explored, including trade-offs of dietary preferences against nutrient quality and GHGE. All modelled diets had improved adherence to FBDG and had either the least deviation from the observed diet (MaxP, for the most preferred diet), the highest nutrient quality (MaxH, for the healthiest diet) or the lowest GHGE (MaxS, for the most environmentally sustainable diet). Modelled diets were compared with observed diets for dietary preferences, nutrient quality, environmental impact and food and nutrient composition.

In order to characterise dietary preferences, we used the minimum deviation (MINDV) approach ${ }^{(12)}$, which minimises the sum of positive and negative deviations (absolute values) of food group intake from the observed diet. For interpretation purposes, we used a so-called diet similarity index to serve as a simple description of the overall similarity between the observed diet and the alternative healthier diet. For each individual, this diet similarity index was calculated as the summed amount of each food group that remains the same in the modelled diet as compared with the observed diet divided by total diet weight of the observed diet. In order to characterise nutrient quality and environmental impact of the diet, we used NRD15.3 $3^{(34,35)}$ and GHGE, respectively. NRD15.3 is the unweighted sum of percentage daily values for the fifteen nutrients to encourage (protein, MUFA, dietary fibre, Ca, Fe, K, Zn, vitamins A, D, E, C, $\mathrm{B}_{1}, \mathrm{~B}_{2}, \mathrm{~B}_{12}$ and folate) minus the sum of percentage maximum recommended values for the three nutrients to limit (saturated fat, added sugar and $\mathrm{Na}$ ). The NRD15.3 was calculated 10.460 MJ $(2500 \mathrm{kcal})$ for men and $8.368 \mathrm{MJ}(2000 \mathrm{kcal})$ for women 
and capped at $100 \%$ of the dietary value. Due to the slight between-country differences in the definition of $\mathrm{Na}$ and added sugar, the NRD15.3 was not entirely comparable between the countries. Therefore, we expressed the results relative to the observed diet in strata of country and gender and calculated averages by country. The trade-offs were done by first giving full weight to dietary preferences in the MaxP model (i.e. minimum deviation form observed diet) followed by a $10 \%$ stepwise increase of the weight for either nutrient quality or GHGE. After each step, the alternative diet was calculated until full weight was given to either nutrient quality (MaxH) or GHGE (MaxS).

\section{Results}

\section{Identification of efficient diets}

Table 2 shows the general characteristics of subjects in the study sample and those with efficient diets. The proportion of subjects with an efficient diet ranged from $23 \%$ (Italian women) to $45 \%$ (Czech women). General characteristics were similar to the overall sample for age, educational level and overweight.

\section{Trade-off of dietary preferences against nutrient} quality and environmental sustainability

Figure 1 shows the trade-off of dietary preferences against nutrient quality (1a) and GHGE of the diet (1b) for all four countries averaged for men and women. Of course, the MaxP diet remained closest to the observed diet (diet similarity index on average $85 \%$ ) as compared with the MaxH diet and the MaxS diet (diet similarity index on average 72 and $73 \%$, respectively). Given the FBDG-based modelling, the MaxP diet had already a 4-9\% higher NRD15.3, whereas GHGE was only $0 \cdot 5-5 \%$ lower than the observed country-specific diets. For the MaxH diet, nutrient quality was increased at the expense of diet similarity, and the NRD15.3 increased by 11-20\% (Fig. 1a). In this diet, the GHGE was not sensitive to this trade-off except in Denmark in which this lowered to about $10 \%$. For the trade-off of dietary preferences against GHGE (Fig. 1b), the MaxS diet had a 13-28\% lower GHGE. The NRD15.3 appeared sensitive to this trade-off and subsequently increased by $6-12 \%$. The shape of the trade-off curves for nutrient quality and GHGE shows that the largest gains occurred in the first part of the curve and were attenuated thereafter. Moreover, the $\mathrm{MaxH}$ diet only marginally affected GHGE, and the MaxS diet only reached half the maximum for nutrient quality, therefore indicating a trade-off between these objectives.

Food composition of observed and modelled diets

Figure 2 presents the total weight of the diet and the amounts consumed for each main food group for the

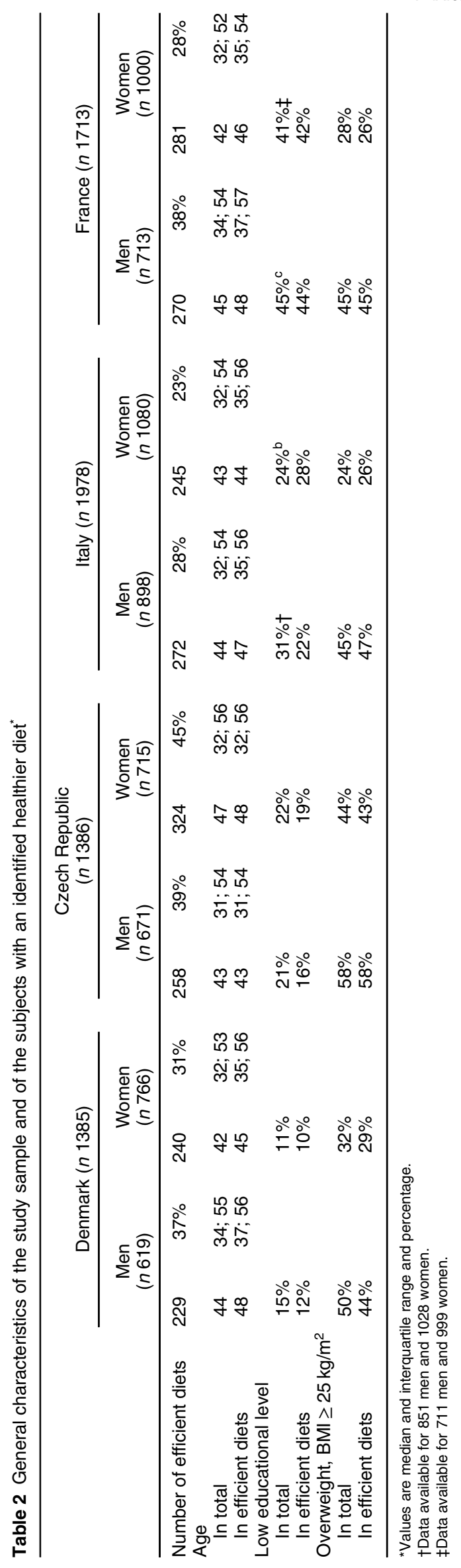


(A)

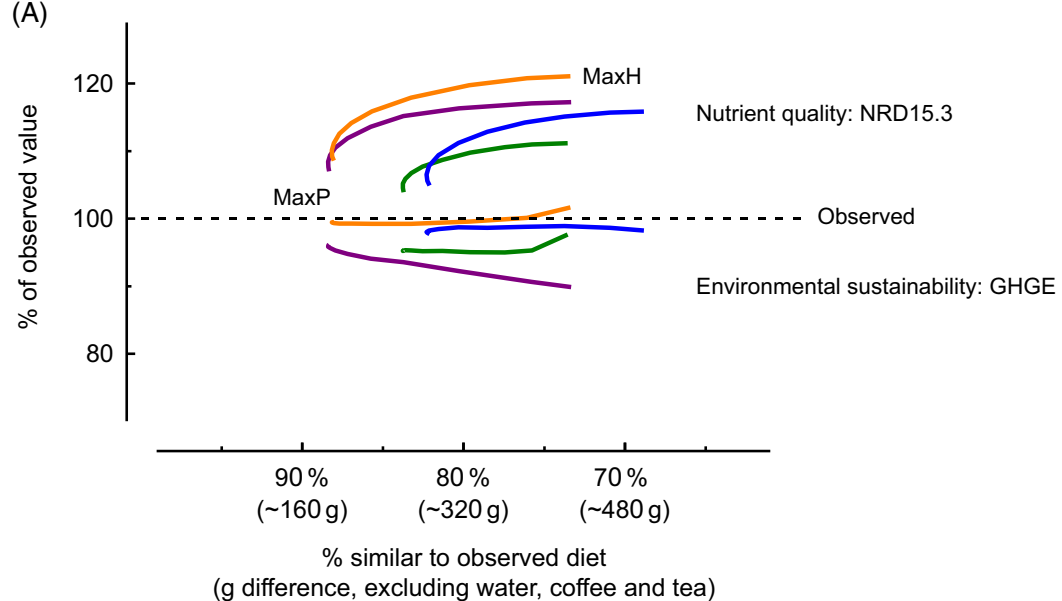

(B)

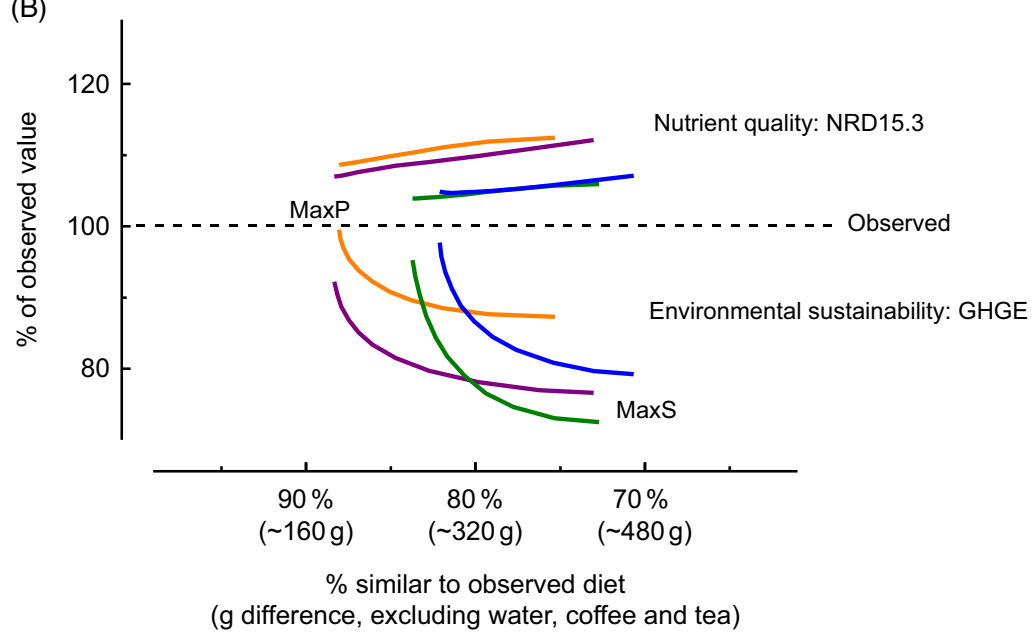

Fig. 1 Trade-offs of dietary preferences ${ }^{a}$ against nutrient quality $(A)$ and environmental sustainability of the $\operatorname{diet}^{c}(B)^{\text {d.a }}$ Dietary pref- $^{b}$ erences were expressed as the diet similarity index, i.e. weight of foods in the modelled diet that corresponds to the observed diet, as a percentage of the latter (horizontal axis). Total observed food weight (excluding water, coffee and tea) was around $1800 \mathrm{~g} / 2500 \mathrm{kcal}$

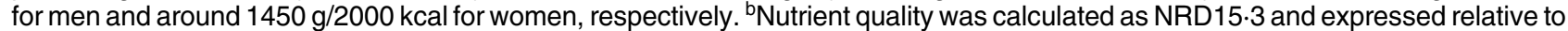
its observed value for each population group (as \%; upper part of the vertical axis). Observed NRD15.3 was 938 for Denmark, 812 for Czech Republic, 977 for Italy and 831 for France. ${ }^{\circ}$ Environmental sustainability of the diet used GHGE as indicator and is expressed relative to its observed value (as \%: lower part of the vertical axis). Observed GHGE in kgCO2eq/2000 kcal was 4.85 for Denmark, 4.42 for Czech Republic, 4.88 for Italy and 6.08 for France. ${ }^{d}$ When relaxing dietary preferences, the upper part of the graphs shows the relative changes in nutrient quality (using NRD15.3) of the improved diets and the lower part of the graphs the relative changes in environmental sustainability (using GHGE). Figure 1a depicts the trade-off between dietary preferences and nutrient quality, i.e. maximising NRD15.3 while relaxing the criterion for diet similarity ( $x$-axis). At the left full weight is given to diet similarity (MaxP) and at the right full weight is given to nutrient quality $(\mathrm{MaxH})$, results for NRD15.3 are depicted at the upper part of Figure 1a and for GHGE at the lower part of Figure 1a. In a similar way, Figure $1 \mathrm{~b}$ depicts the results on NRD15.3 and GHGE when minimising GHGE and relaxing the criterion for dietary preferences, with at the right full weight is given to GHGE (MaxS). To convert kcal to kJ multiply it by 4.184. Denmark, —, Czech Republic, —, Italy, —, France

observed and modelled diets (see also Supplementary Table 1)

In all countries and for both genders, total weight of foods in the diet (including dairy and excluding coffee, tea, water, sweet and alcoholic beverages) was higher for the modelled diets. For the MaxP diet, this amounted to a higher diet weight of $65-130 \mathrm{~g} / 10.460 \mathrm{MJ}$ (2500 kcal) (6-9\% increase) for men and 60-140 g/8.368 MJ (2000 kcal) (6-11\%) for women. This was followed by the MaxS diet in which diet weight was around 122-288 g/10.460 MJ (2500 kcal) (11$20 \%$ ) higher for men and 106-248 g/8.368 MJ (2000 kcal)
(11-21\%) higher for women as compared with observed. The MaxH diet had the highest amount of foods, which was around $240-310 \mathrm{~g} / 10 \cdot 460 \mathrm{MJ}(2500 \mathrm{kcal})(18-24 \%)$ higher for men and 211-380 g/8.368 MJ (2000 kcal) (20$29 \%$ ) higher for women as compared with the observed diet. For drinks (excluding water, coffee and tea), all models showed that sweetened and alcoholic beverages had to be substantially reduced, especially for the MaxH and MaxS diets among men (43-52\% reduction).

Despite the higher food consumption, the proportion of animal-sourced foods remained similar to the observed 
(A)

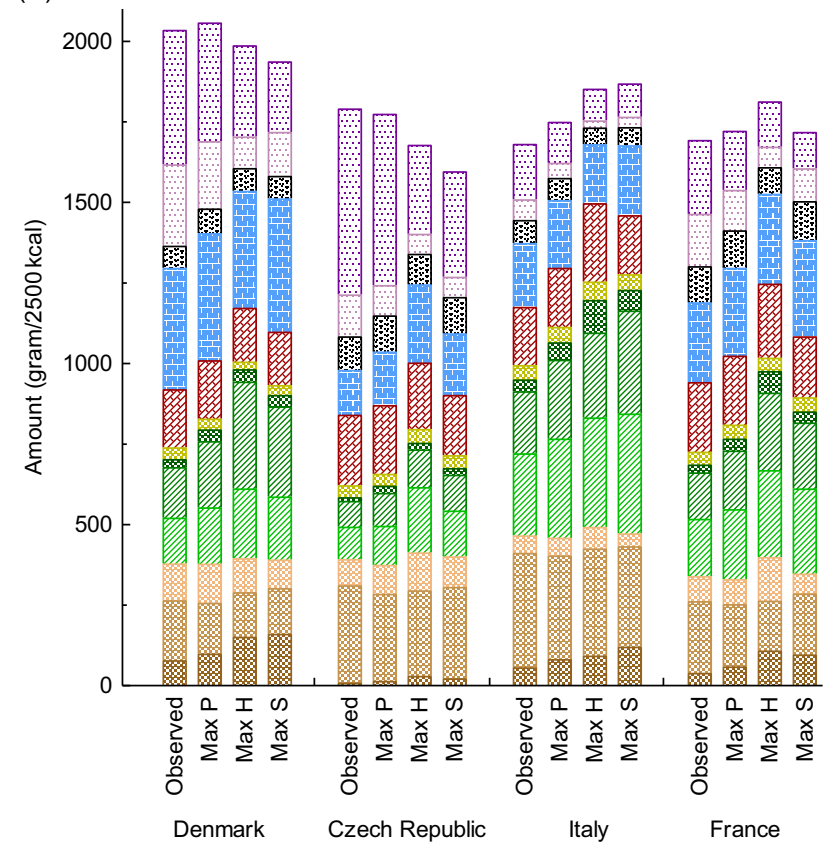

(B)

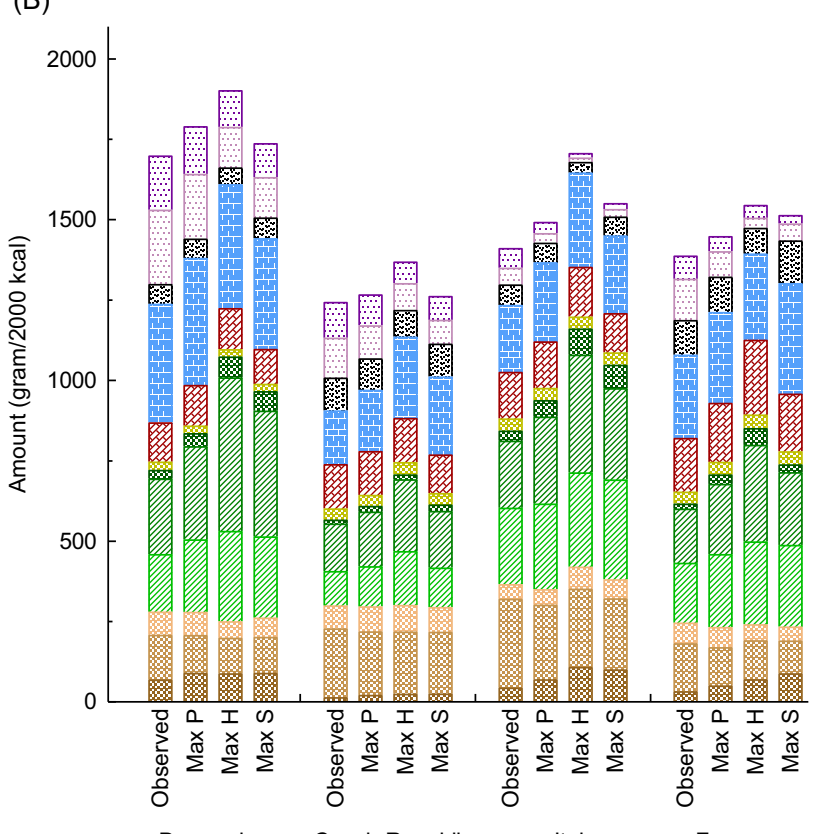

Fig. 2 Mean quantities for main food groups in the observed and modelled diets ${ }^{a, b}$. ${ }^{a}$ All modelled diets improved on the dietary components mentioned in Table 1. MaxP is the most preferred diet based on minimal deviation from the observed diet, MaxH the healthiest diet based on NRD15.3 for nutrient quality and MaxS the most environmentally sustainable diet based on GHGE. 'Information on amount of coffee, tea and water in the diets is not included in the graph. To convert kcal to kJ multiply it by

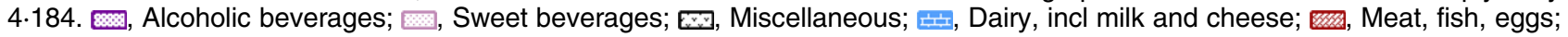

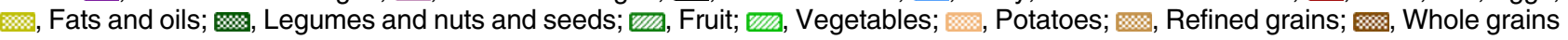

diets for all modelled diets. In particular, approximately $35 \%$ of total weight (including dairy and excluding water, coffee and tea) is animal sourced. However, there were shifts within the animal- and plant-sourced food groups with, as expected, most marked shifts for the food groups that are included in the FBDG and thus used by the model for selecting efficient diets. The total amount of animalsourced foods was higher in most modelled diets, except for MaxH in Danish men and MaxS in Danish women (Fig. 3). The amount of meat from beef and pork was, however, lower in all modelled diets. The amount of poultry remained roughly similar, but amounts of fish, eggs and total dairy were higher in most of the modelled diets. Total dairy products (including cheese) were not entered in the model and amounts decreased or increased slightly ( -8 to $+74 \%$ ) depending on the objectives of the model and population subgroup. Taken together, for the MaxP diet, animal-sourced foods were on average $25 \mathrm{~g}$ higher for men $(+5 \%)$ and $40 \mathrm{~g}$ higher for women $(+8 \%)$ as compared with animal-sourced foods in the observed diet. Amounts were even higher for the MaxH diet for men (up to $60 \mathrm{~g}(+16 \%)$, except for Danish men) and for the MaxS diet for Czech and French women (up to $79 \mathrm{~g}(+21 \%))$.

The total amount of plant-sourced foods was also higher in all modelled diets (11-36\%). In particular, vegetables $(+36 \%)$, fruits $(+49 \%)$, legumes $(+91 \%)$ and whole grains $(+103 \%)$ increased, whereas refined grains decreased
$(-16 \%)$ as most clearly seen in the MaxH diet and the MaxS diet. The amount of nuts and seeds was only slightly higher than observed.

Results of the modelled diets differed by country and gender and were dependent on the trade-offs between dietary preferences, nutrient quality and GHGE. For most population groups, the MaxH diet had the highest amount of fruit ( $+60 \%$, except for Italian men), vegetables $(+75 \%$, except for Italy), legumes $(+132 \%)$ and fish $(+124 \%)$ and the lowest amount of sweetened beverages ( $-60 \%$, except for Czech women) as compared with the other diets. The amount of red and processed meat was lowest for the MaxS diet (-45\%) followed by the MaxH diet (-36\%) and was closest to the observed diets for the MaxP diet $(-20 \%)$ (see Supplementary Table 1$)$.

\section{Nutrient quality of observed and modelled diets}

In detail for the nutrient quality, the nutrient evaluations for each nutrient included in the NRD15.3 are shown in Supplementary Table 2 . In our data, the three modelled diets alleviated the nutrient inadequacies. Nevertheless, the average intakes remained below recommended intake levels for dietary fibre, $\mathrm{K}, \mathrm{Mg}$, vitamin $\mathrm{E}$ and vitamin $\mathrm{D}$ although there were differences by modelled diet, country and gender. Nutrient inadequacies showed the most improvement for a MaxH diet and the least for a MaxP diet. Next to the nutrients to encourage, the NRD15.3 included 
Benchmarking diets for health and environment

(A)

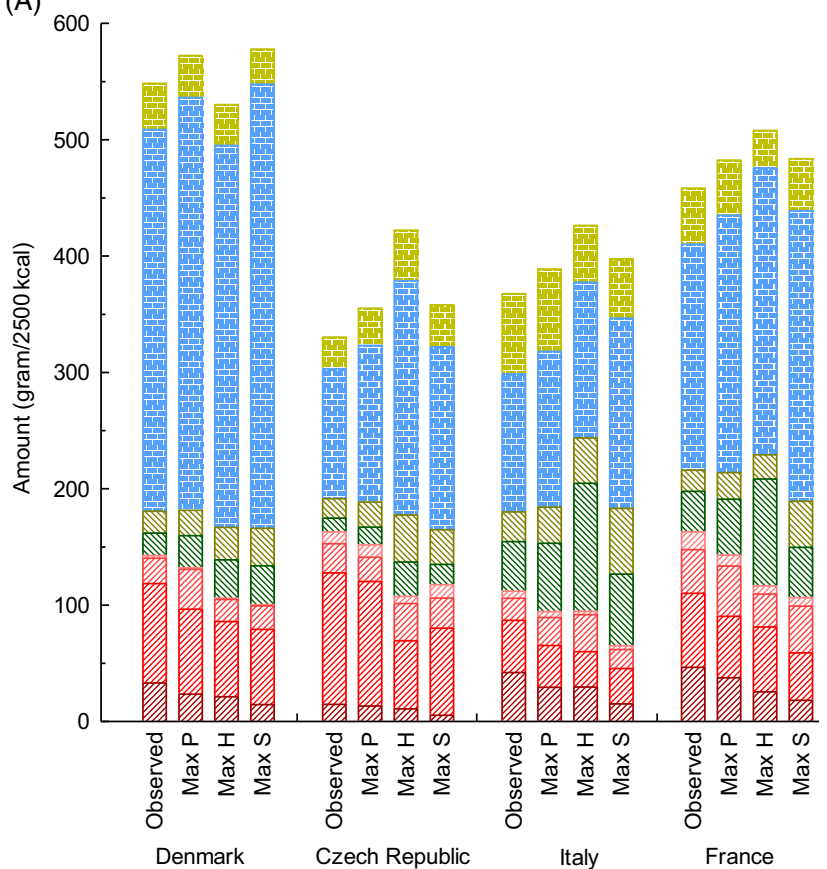

(B)

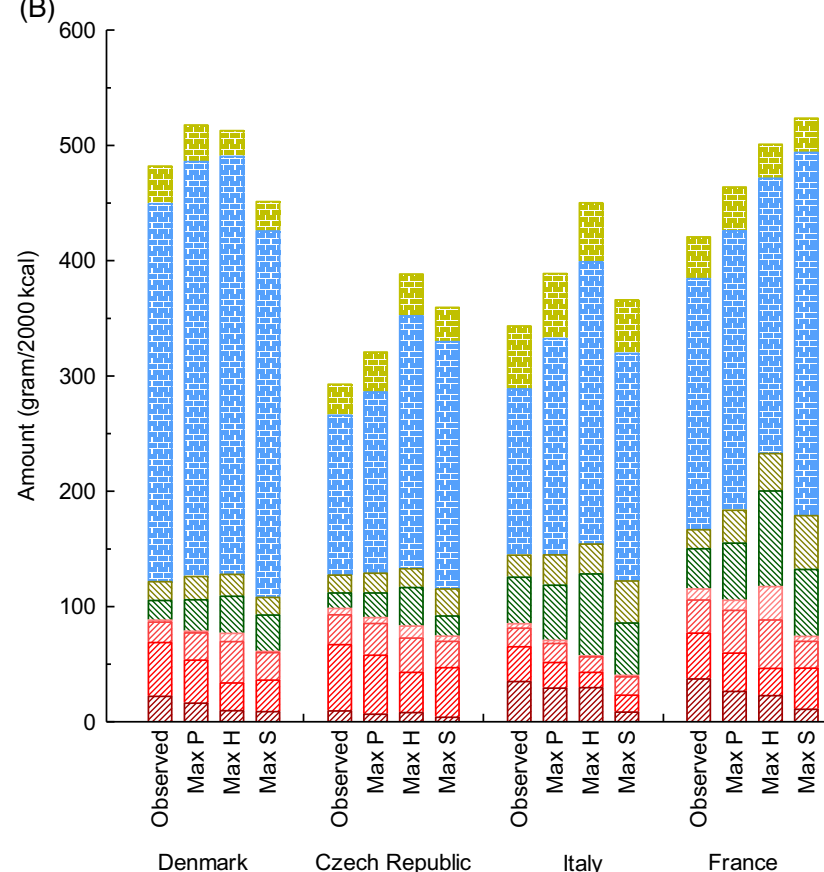

Fig. 3 Mean quantities for main animal-sourced food groups in the observed and modelled diets ${ }^{a}$. ${ }^{a}$ All modelled diets improved on the dietary components mentioned in Table 1 . MaxP is the most preferred diet based on minimal deviation from the observed diet, MaxH the most healthy diet based on NRD15.3 for nutrient quality, and MaxS the most environmentally sustainable diet based on GHGE. To convert kcal to kJ multiply it by 4.184. 푸, Cheese; 포, Milk etc.; 제, Eggs;

three nutrients to limit. Compared with the observed diet, intakes in the modelled diets were improved (i.e. lower) for saturated fat in all four countries and for added sugar in Denmark and Czech Republic, while intakes remained roughly the same for $\mathrm{Na}$ in all four countries and for added sugar in Italy and France. A note of caution is due here given that $\mathrm{Na}$ and added sugar were assessed differently in the countries. For example, in Italy, only the $\mathrm{Na}$ intake from raw foods was included, which resulted in a Na intake that was much closer to or even lower than the maximum reference value. In Italy and France, total sugar was assessed that resulted in less change as compared with added sugar that excluded sugars naturally occurring in fruit, vegetables and dairy.

\section{Discussion}

This application of the DEA model to dietary survey data from four European countries of Denmark, Czech Republic, Italy and France showed that the most preferred diet had a larger impact on nutrient quality (on average $6 \%$ higher) than on GHGE (on average $4 \%$ lower). Interestingly, however, the diet with the highest nutrient quality (on average 16\% higher NRD15.3) did not result in a lower GHGE. Yet, the most environmentally sustainable diet (on average $21 \%$ less GHGE) had a higher nutrient quality (on average $9 \%$ higher NRD15.3) as compared with the observed diet. Although the results differed by country and gender, the modelled diets had higher amounts of both plant- and animal-sourced foods but with the relative amounts of foods remaining similar. Diets of the highest nutrient quality exhibited the highest amounts of plant-sourced foods, while diets of the lowest GHGE contained the lowest amounts of red and processed meat.

The modelled diets accounted for the prevailing dietary preferences of the study populations. This was because the DEA model preserves existing interrelationships among food groups as this model uses a linear combination of observed diets as benchmarks. These observed diets implicitly accounted for sensory preferences and culinary practices as well as the availability, acceptability and affordability of the diet. We stratified our analyses by country and gender under the assumption that subjects in these strata share many unspecified variables, including educational level, overweight status and determinants of food choice. Indeed, the descriptive variables of the efficient diets were comparable with their representative population segments (Table 2). In our data, nutrient quality but not environmental impact of the diet was associated with gender and educational level ${ }^{(17,27)}$. Future analyses should consider, however, also accounting for socio-economic status or include indicators such as sensory profiles ${ }^{(36,37)}$ or food prices ${ }^{(38)}$. Such analyses in a more homogeneous population subgroup could identify solutions for dietary improvement that fit even better with subgroup-specific dietary 
practices and preferences. In this way, the DEA model keeps the proposed diets realistic as they stay within the range of observed national diets.

In order to derive the most preferred diet, the DEA model minimised the absolute value of deviation between the modelled and observed food intake summed over all food groups. Although this algorithm can be further improved by, for example, using relative or squared difference instead and/or modelling food replacements within meals and recipes instead of day menus, it is important to realise that such an algorithm of minimal deviation is needed for modelling more feasible and realistic dietary improvement options. Importantly, our results suggested that a partial shift to poultry, fish and increased intake of legumes would be preferable to a sole focus on fully reducing red and processed meat. This partial shift is in line with the food choices of a growing number of consumers identifying themselves as meat reducers or flexitarians ${ }^{(39)}$, which confirms the feasibility of adopting our proposed diets. Potential strategies that consumers adopt for eating less meat include meatless days with or without meat substitutes, the promotion of smaller portions of meat and if possible in combination with more sustainable produced meat and/or larger portion of fruit and vegetables ${ }^{(39-41)}$. In this way, the DEA model provides a necessary first step to arrive at more feasible and realistic changes and thus could be used to guide national policies towards improved health and environmental impact.

Data comparability between the countries was a challenge because food consumption data were obtained from different national dietary surveys. In order to enhance comparability, we expressed nutrients and GHGE relative to energy intake ${ }^{(17,27)}$ and used the NRD15.3 as a summary measure for nutrient quality. When the NRD15.3 was used to maximise nutrient quality, protein-rich foods, such as fish, eggs, dairy, legumes and nuts and seeds, were likely to be chosen as a meat replacement. This was because of the inclusion of protein, vitamins $\mathrm{B}_{1}, \mathrm{~B}_{2}$ and $\mathrm{B}_{12}, \mathrm{Fe}$ and $\mathrm{Zn}$ as nutrients to encourage in the NRD15.3. However, as $\mathrm{Na}$ and added sugar are part of nutrients to limit in the NRD15.3, cross-country comparisons were hampered as they were assessed differently in the countries. We therefore expressed our results relative to the observed diet in each of the strata. For example, in Italy and France, total sugar was assessed which biased the NRD15.3 downward as compared with Denmark or Czech Republic in which only added sugar was assessed. This may partially explain why in Italy intakes of fruit, vegetables and whole grains were not the highest for the diet that maximised NRD15.3. In Italy, only Na intake from raw foods was assessed, which biased the NRD15.3 upward as compared with Denmark, Czech Republic and France in which discretionary salt was assessed as well. Furthermore, environmental impact only included GHGE data averaged for the European context, and further refinement to national food systems would be needed to incorporate differences between agricultural systems, including the influence of locally produced food and seasonality ${ }^{(42)}$. Our use of average footprints for the four countries rather than country-specific estimates may have partially masked the potential role of food production systems relative to dietary patterns; however, incorporating different food systems would also require incorporation of food trade and prices, which is beyond the scope of this diet model. Moreover, pan-European standardised indicators of land and freshwater use, nitrogen and phosphorus flows and biodiversity could give a more balanced picture of environmental impact of the diet. Given these imperfections in comparability of survey data and incompleteness of indicators, the observed and modelled diets differed by country. And, yet, the general pattern was similar in both men and women, thus emphasising the robustness of these findings.

The food consumption data were derived from national dietary surveys, and we used the average of two nonconsecutive days for each individual which slightly reduces day-to-day variability ${ }^{(43)}$. The use of two averaged days for the benchmark diets exploited within- and betweensubject variation within the demographic strata and created a larger window of opportunity for improving diets than time-integrated long-term dietary habits. Since modelled diets are combinations of observed diets, the modelled range of food and nutrient intake and GHGE was slightly lower than that of the observed diets. Nevertheless, the range of solutions remained in the same order of magnitude (data not shown), which suggests that the results from the three models were realistic steps for dietary shifts at the population level. At the same time, the use of only $2 \mathrm{~d}$ raised questions on foods consumed only occasionally, such as fish, nuts and seeds and legumes. Fish was of particular concern given its recommended consumption frequency is around one to two portions a week in order to avoid toxicological risks because of contaminants like (methyl)mercury ${ }^{(44)}$. Using this occasionally consumed, high portion size of fish as a benchmark for an average diet would shift the whole population to these high intakes, which are ultimately unrepresentative of a typical day. This was tackled by first capping fish intake at $1 / 7^{\text {th }}$ of one portion to avoid favouring higher intakes than recommended and second by replacing upper intake levels by a lowest non-zero intake divided by two in order to disadvantage extreme upper intakes all while benchmarking diets. Given their small portion sizes and infrequent consumption, legumes and nuts and seeds did not increase substantially in our diets. In other studies, food-based linear programming approaches have shown that these food groups can contribute to healthy and environmentally sustainable $\operatorname{diet}^{(7,45-47)}$ as was also suggested by the healthy reference diet presented by the EAT-Lancet Commission $^{(48)}$. Targeted efforts and product development 
would therefore be warranted in order to increase consumption of some foods beyond current national eating habits.

Results of our analyses depend on the choice of variables included in the model. In our modelling strategy, we aimed for diets that would increase adherence to FBDG that are considered relevant to non-communicable disease risk (factor) reduction (see Table 1). After all, a healthy diet not only implies meeting FBDG but also includes nutrient requirements, non-nutrients, bio-actives and direct physiological effects on hunger, digestion and satiation. In light of the underlying nutritional rationale, we replaced the guideline 'use oils instead of hard fats' by unsaturated and saturated fats as 'to increase' and 'to decrease', respectively. In all population groups, the modelled diets performed the same or better for all nutrients, except in women from the Czech Republic. In these women, a substantial decrease in animal-sourced foods occurred which subsequently lowered intakes of Ca, Zn, vitamin $\mathrm{B}_{2}$ and vitamin $\mathrm{B}_{12}$ (results not shown). In order to safeguard the intakes of these nutrients, these nutrients were then added to the DEA model. Nevertheless, it must be realised that the modelled diets are based on calculated nutrient intake from dietary surveys that do not account for bioavailability. We observed that the proportion of animalsourced foods and the daily protein intake in modelled diets was essentially similar to observed diets. However, bioavailability of protein and some minerals from plantsourced foods is less than from animal-sourced foods; thus, warranting physiological research into nutrients that can become critical for vulnerable population groups. For example, some plant compounds can inhibit the absorption of minerals, such as $\mathrm{Ca}, \mathrm{Zn}$ and non-haem $\mathrm{Fe}^{(49,50)}$, whereas vitamin $\mathrm{C}$ may increase the bioavailability of $\mathrm{Fe}$ from plant foods ${ }^{(49)}$.

In line with previous studies ${ }^{(47,51-55)}$, the transition to a healthier diet implies increasing the amounts of fruits, vegetables and whole grains, whereas an environmentally more sustainable diet requires to prioritise lowering red and processed meat and only mildly increasing legumes, nuts and seeds. Although results differed slightly by country and gender, dairy products essentially remained in the diet. However, caution must be applied when decreasing beef intake while keeping dairy intake similar. As when such diets are implemented on a wider scale, the amount of beef meat proposed should be in correspondence with the meat production volumes produced as a co-product from milk production. Food system model that account for food chain interdependency suggests that in animal husbandry of dairy cows, $1 \mathrm{~g}$ of beef can be produced for every $46 \mathrm{~g}$ of milk that is produced ${ }^{(56-59)}$.

Nevertheless, the proportion of animal- and plantsourced food in the modelled diets remained similar, but nutrient quality (NRD15.3) increased. Surprisingly, the total amount of foods (including dairy) increased by 5-30\% for the modelled diets, mostly for the healthiest diet, and thus the overall energy density for these food groups decreased accordingly. At the same time, we observed that the modelled healthier and more environmentally sustainable diets had lower amounts of sweetened and alcoholic beverages, especially in men. These results show that at the national level, important first steps can be made in nutritional policy, but the priorities will affect nutrient quality and environmental sustainability differently. However, on average, the proposed diets do not achieve the proposed global targets for healthy and environmentally sustainable diets and additional policy measures are thus warranted.

In conclusion, the DEA benchmarking diet model shows that generally accepted FBDG and nutrient requirements can be used to model healthier and more environmentally sustainable diets based on dietary surveys from a set of diverse European diets. While improving the adherence to FBDG, the diet with the largest improvement in GHGE resulted in a win-win for both health and the environment, but did not achieve the full health potential. Focusing on nutrient quality alone, however, did not improve GHGE. As such, larger dietary changes and complementary measures are required in agricultural production and processing $^{(60)}$, food loss and waste management, as well as an equitable distribution via the food supply chain ${ }^{(61)}$.

\section{Acknowledgements}

Acknowledgements: Not applicable. Financial support: This work was supported by the European Union's H2020 Programme under grant Agreement number 633692 (SUSFANS: Metrics, models and foresight for European sustainable food and nutrition security) and TiFN under Project Agreement number 15SD01 (SHARP-BASIC). A.K., J.M.G. and P.v.V. received research funding from TiFN (grant 15SD01_SHARP). Conflicts of interest: For the sake of clarity, it should be mentioned that E.T. has been involved in small-grant research projects based on funding from the industry (Danish Dairy Research Foundation and Danish Agriculture and Food Council). Availability of data and materials: The SHARP Indicators Database (SHARP-ID) used to calculate the diet-related environmental impact is available in a data repository; doi: https://doi.org/ 10.17026/dans-xvh-x9wz. Restrictions apply to the availability of the data that support the findings of this study, which were used under license for this study, and so are not publicly available. Authorship: P.v.V. and J.M.G. designed the research; M.D., L.M., L.D.A., A.T., C.D., S.H. and E.T. were responsible for dietary data collection; E.M. analysed data and received input for the diet modelling from A.K.; E.M., P.v.V., J.M.G., A.K. and S.B. were responsible for data interpretation; E.M. wrote the manuscript, which was reviewed by all authors for intellectual content. All authors read and approved the final version of the manuscript. Ethics of human subject participation: The national dietary 
survey of Denmark (DANSDA 2005-2008), Czech Republic (SISP04) and Italy (INRAN-SCAI 2005-2006) did at that time not need to notified and endorsed by the Ethical Committee. The national dietary survey of France (INCA2 study 2006-2007) was approved by the French Data Protection Authority (Commission Nationale Informatique et Libertés).

\section{Supplementary material}

To view supplementary material for this article, please visit https://doi.org/10.1017/S1368980020003341

\section{References}

1. Forouzanfar MH, Alexander L, Anderson HR et al. (2015) Global, regional, and national comparative risk assessment of 79 behavioural, environmental and occupational, and metabolic risks or clusters of risks in 188 countries, 1990-2013: a systematic analysis for the Global Burden of Disease Study 2013. The Lancet 386, 2287-2323.

2. Vermeulen SJ, Campbell BM \& Ingram JSI (2012) Climate change and food systems. Ann Rev Environ Res 37, 195.

3. Tubiello F, Salvatore M, Cóndor Golec R et al. (2014) Agriculture, Forestry and Other Land Use Emissions by Sources and Removals by Sinks. Rome: Statistics Division, Food and Agriculture Organization.

4. Steinfeld H, Gerber P, Wassenaar T et al. (2007) Livestock's Long Shadow. Rome: Environmental issues and options FAO.

5. Buttriss JL, Briend A, Darmon N et al. (2014) Diet modelling: how it can inform the development of dietary recommendations and public health policy. Nutr Bull 39 $115-125$.

6. Gazan R, Brouzes CMC, Vieux F et al. (2018) Mathematical optimization to explore tomorrow's sustainable diets: a narrative review. Adv Nutr 9, 602-616.

7. Ferrari M, Benvenuti L, Rossi L et al. (2020) Could dietary goals and climate change mitigation be achieved through optimized diet? The experience of modeling the national food consumption data in Italy. Front Nutr 7, 48.

8. Dantzig GB (1990) The diet problem. INFORMS J Appl Anal 20, $43-47$.

9. van Dooren C (2018) A review of the use of linear programming to optimize diets, nutritiously, economically and environmentally. Front Nutr 5, 48.

10. Mertens E, van't Veer P, Hiddink GJ et al. (2017) Operationalising the health aspects of sustainable diets: a review. Pub Health Nutr 20, 739-757.

11. Smith VE (1959) Linear programming models for the determination of palatable human diets. Am J Agr Econ 41, 272-283.

12. Kanellopoulos A, Gerdessen JC, Ivancic A et al. (2020) Designing healthier and acceptable diets using data envelopment analysis. Publ Health Nutr 23(13), 1-13.

13. Pedersen A, Fagt S, Groth M et al. (2009) Danskernes kostvaner 2003-2008 (The Danish Food Consumption Survey). Søborg, Denmark: DTU Fødevareinstituttet (Food Institute).

14. Ruprich J, Dofkova M, Rehurkova I et al. (2006) Individual food consumption: the national study SISP04 $\mathrm{CHFCH}$ National Institute of Public Health in Prague: CHFCH NIPH in Prague. http://www.chpr.szu.cz/spotrebapotravin.htm (accessed September 2017).
15. Leclercq C, Arcella D, Piccinelli R et al. (2009) The Italian national food consumption survey INRAN-SCAI 2005-06: main results in terms of food consumption. Publ Health Nutr 12, 2504-2532.

16. Agence Française de Sécurité Sanitaire des Aliments (AFSSA) (French Food Safety Agency) (2009) Synthèse de l'étude individuelle nationale des consommations alimentaires 2 (INCA 2), 2006-2007 (Report of the 2006/2007 Individual and National Study on Food Consumption 2 (INCA 2)). pp. 1-44. Maisons-Alfort Cedex: Direction de l'évaluation des risques nutritionnels et sanitaires (Department of Nutritional and Health Risk Assessment).

17. Mertens E, Kuijsten A, Dofkova M et al. (2018) Geographic and socioeconomic diversity of food and nutrient intakes: a comparison of four European countries. Europ J Nutr 58(4), 1475-1493.

18. EFSA (European Food Safety Authority) (2015) The food classification and description system FoodEx2 (revision 2). Parma, Italy: EFSA supporting publication En-804:90.

19. EFSA (European Food Safety Authority) (2011) Use of the EFSA comprehensive European food consumption database in exposure assessment. EFSA J 9, 2097.

20. Fødevareinformatik (Food Informatics) (2005) Fødevaredatabanken (Food Composition Database), Version 6.0. http://frida.fooddata.dk (accessed September 2017).

21. Fødevareinformatik (Food Informatics) (2008) Fødevaredatabanken (Food Composition Database), Version 7. http://www.foodcomp.dk/ (accessed September 2017).

22. Institute of Agricultural Economics and Information (2016) Czech Food Composition Database Version 6.16. http:// www.nutridatabaze.cz/ (accessed September 2017).

23. Slovak Food Composition Data Bank, Department of Risk Assessment Food Composition Data Bank and Consumer's Survey VUP Food Research Institute (2016). http://www. pbd-online.sk/ (accessed September 2017).

24. Istituto Nazionale di Ricerca per gli Alimenti e la Nutrizione (National Research Institute for Food and Nutrition), Banca Dati di Composizione degli Alimenti (Food Composition database) (2016). http://nut.entecra.it/646/tabelle_di_ composizione_degli_alimenti.html (accessed September 2017).

25. Institut national de la recherche agronomique (National Institute of Agronomic Research) (1995) Répertoire général des aliments (General Inventory of Foods). Technique $\varepsilon$ Documentation - Lavoisier. Paris, France: Lavoisier.

26. French Food Safety Agency (AFSSA) (2008) French Food Composition Table, Version. http://www.afssa.fr/Table CIQUAL/index.html (accessed September 2017).

27. Mertens E, Kuijsten A, van Zanten HHE et al. (2019) Dietary choices and environmental impact in four European countries. J Clean Prod 237, 117827.

28. Mertens E, Kaptijn G, Kuijsten A et al. (2019) SHARPindicators database towards a public database for environmental sustainability. Data Brief 27, 104617.

29. Willett WC, Howe GR \& Kushi LH (1997) Adjustment for total energy intake in epidemiologic studies. Am J Clin Nutr $\mathbf{6 5}$, 1220S-1228S.

30. Goldberg G, Black A, Jebb S et al. (1991) Critical evaluation of energy intake data using fundamental principles of energy physiology: 1. derivation of cut-off limits to identify underrecording. Europ J Clin Nutr 45, 569-581.

31. Black AE (2000) Critical evaluation of energy intake using the Goldberg cut-off for energy intake: basal metabolic rate. A practical guide to its calculation, use and limitations. Int $J$ Obes Relat Metab Dis 24, 1119-1130.

32. Cooper WW, Seiford LM \& Kaoru T (2007) Data Envelopment Analysis. New York: Springer. 
33. Zhu J \& Cook WD (2007) Modeling Data Irregularities and Structural Complexities in Data Envelopment Analysis. New York: Springer Science \& Business Media.

34. Fulgoni VL 3rd, Keast DR \& Drewnowski A (2009) Development and validation of the nutrient-rich foods index: a tool to measure nutritional quality of foods. J Nutr 139, 1549-1554.

35. Drewnowski A (2009) Defining nutrient density: development and validation of the nutrient rich foods index. $J \mathrm{Am}$ Coll Nutr 28, 421S-426S.

36. van Langeveld AWB, Teo PS, de Vries JHM et al. (2018) Dietary taste patterns by sex and weight status in the Netherlands. Brit J Nutr 119, 1195-1206.

37. van Bussel LM, Kuijsten A, Mars M et al. (2019) Taste profiles of diets high and low in environmental sustainability and health. Food Qual Prefer 78, 103730.

38. Wilson N, Nghiem N, Mhurchu C et al. (2013) Foods and dietary patterns that are healthy, low-cost, and environmentally sustainable: a case study of optimization modeling for New Zealand. PLoS One 121, 2271-2283.

39. de Bakker E \& Dagevos H (2012) Reducing meat consumption in today's consumer society: questioning the Citizenconsumer gap. J Agr Environ Eth 25, 877-894.

40. de Boer J, Schösler H \& Aiking H (2014) "Meatless days" or "less but better"? Exploring strategies to adapt Western meat consumption to health and sustainability challenges. Appetite 76, 120-128.

41. Verain MC, Dagevos H \& Antonides G (2015) Sustainable food consumption: product choice or curtailment? Appetite 91, 375-384.

42. Notarnicola B, Sala S, Anton A et al. (2017) The role of life cycle assessment in supporting sustainable agri-food systems: a review of the challenges. I Clean Prod 140 , 399-409.

43. Willett W (2012) Nutritional Epidemiology. New York: Oxford University Press.

44. EFSA (European Food Safety Authority) (2015) Statement on the benefits of fish/seafood consumption compared to the risks of methylmercury in fish/seafood. EFSA J 13, 3982

45. Maillot M, Vieux F, Amiot MJ et al. (2010) Individual diet modeling translates nutrient recommendations into realistic and individual-specific food choices. Am J Clin Nutr 91, 421-430.

46. Tyszler M, Kramer G \& Blonk H (editors) (2014) Just eating healthier is not Enoug: studying the environmental impact of different diet scenarios for the Netherlands by linear programming. 9th International Conference LCA of Foods, San Francisco, USA.

47. Green R, Milner J, Dangour AD et al. (2015) The potential to reduce greenhouse gas emissions in the UK through healthy and realistic dietary change. Clim Change 129, 253-265.

48. Willett W, Rockström J, Loken B et al. (2019) Food in the anthropocene: the EAT-Lancet commission on healthy diets from sustainable food systems. The Lancet 393 447-492.

49. American Dietetic Association (2009) Position of the American dietetic association: vegetarian diets. J Am Diet Assoc 109, 1266-1282.

50. Gibson RS, Bailey KB, Gibbs M et al. (2010) A review of phytate, iron, zinc, and calcium concentrations in plant-based complementary foods used in low-income countries and implications for bioavailability. Food Nutr Bull 31, S134-S146.

51. Macdiarmid JI, Kyle J, Horgan GW et al. (2012) Sustainable diets for the future: can we contribute to reducing greenhouse gas emissions by eating a healthy diet? Am J Clin Nutr 96, 632-639.

52. Horgan GW, Perrin A, Whybrow S et al. (2016) Achieving dietary recommendations and reducing greenhouse gas emissions: modelling diets to minimise the change from current intakes. Int J Behav Nutr Phys Activ 13, 46.

53. Vieux F, Perignon M, Gazan R et al. (2018) Dietary changes needed to improve diet sustainability: are they similar across Europe? Europ J Clin Nutr 72, 951-960.

54. Perignon M, Masset G, Ferrari G et al. (2016) How low can dietary greenhouse gas emissions be reduced without impairing nutritional adequacy, affordability and acceptability of the diet? A modelling study to guide sustainable food choices. Public Health Nutr 19, 2662-2674.

55. Kramer GF, Tyszler M, Veer PV et al. (2017) Decreasing the overall environmental impact of the Dutch diet: how to find healthy and sustainable diets with limited changes. Public Health Nutr 20, 1699-1709.

56. Bryngelsson D, Wirsenius S, Hedenus F et al. (2016) How can the EU climate targets be met? A combined analysis of technological and demand-side changes in food and agriculture. Food Policy 59, 152-164.

57. Eurostat (2018) Eurostat - Milk and milk product statistics. https://ec.europa.eu/eurostat/statistics- explained/index.php/ Milk_and_milk_product_statistics\#Milk_products (accessed September 2017).

58. Flysjö A, Cederberg C, Henriksson M et al. (2011) How does co-product handling affect the carbon footprint of milk? Case study of milk production in New Zealand and Sweden. Int J Life Cycle Ass 16, 420-430.

59. IDF (2010) The IDF guide to standard LCA methodology for the dairy sector. Bull Int Dairy Fed 445, 1-40.

60. Van Zanten HHE, Van Ittersum MK \& De Boer IJM (2019) The role of farm animals in a circular food system. Global Food Secur 21, 18-22.

61. Rutten M, Achterbosch TJ, de Boer IJ et al. (2016) Metrics, models and foresight for European sustainable food and nutrition security: the vision of the SUSFANS project. Agric Syst 163, 45-57.

62. Forouzanfar MH, Alexander L, Anderson HR et al. (2015) Global, regional, and national comparative risk assessment of 79 behavioural, environmental and occupational, and metabolic risks or clusters of risks in 188 countries, 1990-2013: a systematic analysis for the Global Burden of Disease Study 2013. The Lancet 386, 2287-323.

63. Kromhout D, Spaaij CJ, de Goede J et al. (2016) The 2015 Dutch food-based dietary guidelines. Eur J Clin Nutr $\mathbf{7 0}$, 869-878.

64. EFSA (European Food Safety Authority) (2017) Dietary reference values for nutrients: summary report. EFSA Support Publ e15121, 92.

65. EFSA (Eurepean Food Safety Authority) (2015) Statement on the benefits of fish/seafood consumption compared to the risks of methylmercury in fish/seafood. EFSA J 13, 3982 . 